\title{
Industrial Macromolecular and Small Molecule Crystallography at the Canadian Light Source
}

Denis Spasyuk, Joel Reid, Shaunivan Labiuk, James Gorin, Kathryn Janzen, Michel Fodje, Kiran Mundboth, and Pawel Grochulski Canadian Light Source, Saskatoon, SK

Synchrotron X-ray radiation sources have had a profound impact on the field of macromolecular crystallography, driven by the various genome projects and demand from the pharmaceutical industry. Currently, synchrotron radiation accounts for $\sim 90 \%$ of all structure determinations. Compared with home sources, synchrotrons provide high X-ray spectral range and brilliance, which significantly improves data quality and enables access to multi- and single-wavelength anomalous dispersion (MAD, SAD).

The Canadian Macromolecular Facility (CMCF) at the Canadian Light Source houses the only crystallographic beamlines in Canada which provides access to these types of sources to academic and industrial users. High surface area of the detectors and fast acquisition time combined with automated sample exchange, centering, and data processing make the CMCF one of the most efficient and attractive beamlines for the pharmaceutical industry.

While designed primarily with macromolecules in mind, the $\mathrm{CMCF}$ beamlines allow for an accurate and flexible collection of not only macromolecular compounds but also small molecule data. The above-listed advantages of synchrotron sources allow the CMCF beamlines to resolve the most challenging crystallographic problems even for single crystals too small to be examined using the traditional home source.

Flexible beam collimators allow us to examine multiple crystals on the same crystal mount while tunable X-ray wavelength helps to decipher structures of unknown materials using the anomalous dispersion technique. A combination of these features enables investigation of complex crystal structures with high degrees of disorder as well as modulated structures.

The CMCF and the Industrial Group at the CLS offer ease of access to the macromolecular beamlines with clear intellectual property policies to industrial and academic users from all over the world. 